\title{
Characterization and Source Investigation of Multidrug- Resistant Salmonella Anatum from a Sustained Outbreak, Taiwan
}

\author{
Ye Feng, Yi-Jung Chang, Shih-Chuan Pan, Lin-Hui Su, Hsin-Chieh Li, \\ Hsin-Ping Yang, Min-Jia Yu, Cheng-Hsun Chiu
}

An ongoing outbreak of multidrug-resistant Salmonella enterica serovar Anatum began in Taiwan in 2015. Pork and poultry were identified as vehicles for transmission. Contaminated meat contributed to the high rate of infections among children. Nearly identical Salmonella Anatum strains have been identified in the United Kingdom, the United States, and the Philippines.

N ontyphoidal Salmonella (NTS) is a major cause for foodborne diseases worldwide. In Taiwan, the ambient climate and flourishing pig-raising industry makes NTS infections rampant. As in other countries, salmonellosis was primarily caused by Salmonella enterica serovars Enteritidis and Typhimurium in Taiwan (1), but rare serovars such as Salmonella Goldcoast have appeared in recent years (2). Recommended antimicrobial treatment options for salmonellosis include fluoroquinolones and extended-spectrum cephalosporins (1). However, resistance to these antibiotics has been emerging in many countries, leading to increased disease prevalence, disease severity, and death and the requirement of last-line antimicrobial drugs (e.g., carbapenems) (3-5).

\footnotetext{
Author affiliations: Sir Run Run Shaw Hospital, Zhejiang University School of Medicine, Hangzhou, China (Y. Feng); Institute for Translational Medicine, Zhejiang University School of Medicine, Hangzhou (Y. Feng); Key Laboratory of Microbial Technology and Bioinformatics of Zhejiang Province, Hangzhou (Y. Feng); Division of Pediatric Infectious Diseases, Chang Gung Memorial Hospital, Chang Gung University College of Medicine, Taoyuan (Y.-J. Chang, S.-C. Pan, C.-H. Chiu); Molecular Infectious Disease Research Center, Chang Gung Memorial Hospital, Chang Gung University College of Medicine, Taoyuan, Taiwan (Y.-J. Chang, L.-H. Su, H.-C. Li, H.-P. Yang, M.-J. Yu, C.-H. Chiu)
}

DOI: https://doi.org/10.3201/eid2612.200147
Since 2015, northern Taiwan has seen an increase in Salmonella infections, caused by previously rare Salmonella Anatum. The infections were also reported in central Taiwan, indicating that this outbreak had already prevailed throughout the entire island (6). Co-resistance to ceftriaxone and ciprofloxacin are the main feature of the outbreak clone. Evidence from epidemiologic, laboratory, and supply-chain investigations identified raw pork and poultry as the vehicle for spread of this strain. More important, genomic comparisons against the global public database indicated that this clone has appeared in Europe, Asia, and America. Given the increasing globalization of foodstuffs, these findings prompt an urgent global sharing of whole-genome sequencing (WGS) data to facilitate disease surveillance and early recognition of international foodborne outbreaks $(7,8)$.

\section{The Study}

Chang Gung Memorial Hospital is a main referral hospital for cities in northern Taiwan, including Taipei, New Taipei, and Taoyuan. The population in this region is $\approx 7$ million. In 2012 , the hospital's clinical microbiology laboratory launched a program to monitor the NTS serovars causing human infections. All Salmonella isolates from patients were collected and serotyped. Before 2015, very few Salmonella Anatum isolates were recovered, and most were susceptible to antimicrobial agents. Since then, an increase has been observed, peaking in 2017 (Figure 1, panel A). As of June 2019, a total of 319 nonrepetitive isolates have been identified; of these, $197(61.8 \%)$ isolates were ceftriaxone-resistant (MIC $\geq 2 \mu \mathrm{g} / \mathrm{mL}), 301$ (94.4\%) were ciprofloxacin-resistant (MIC $\geq 0.12 \mu \mathrm{g} / \mathrm{mL}$ ), and $197(61.8 \%)$ were resistant to both. In addition, $292(91.5 \%)$ isolates were resistant to chloramphenic, and 295 (92.5\%) were resistant to trimethoprim/ 


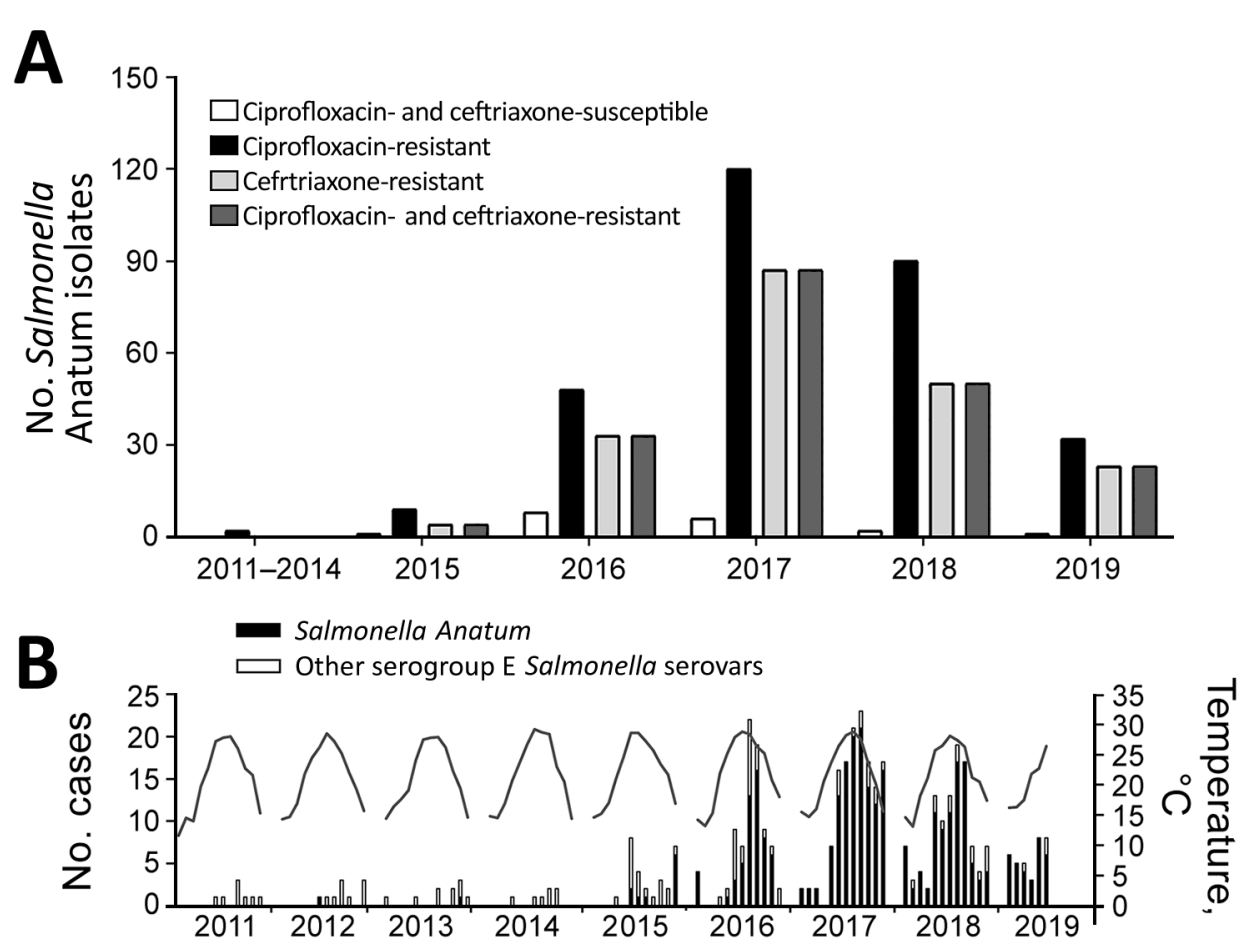

C

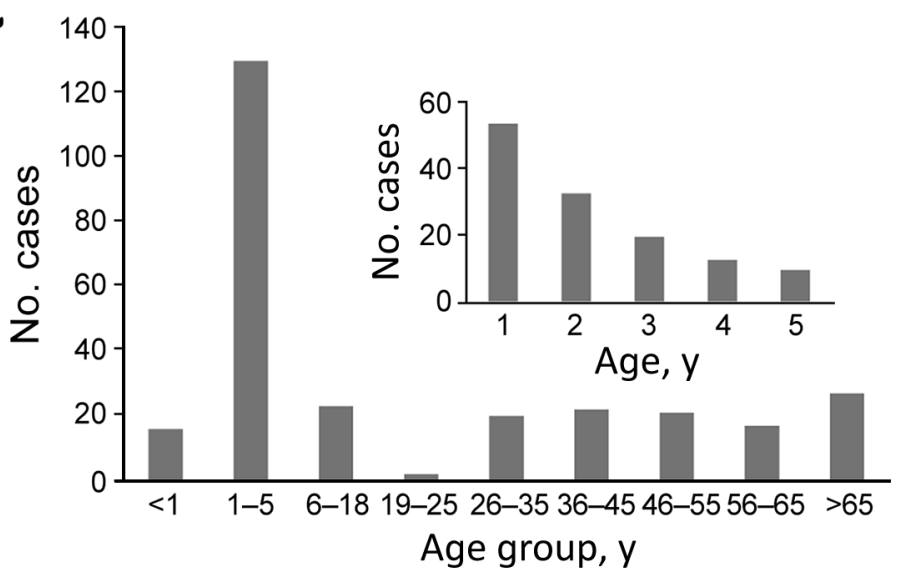

sulfamethoxazole. A positive correlation was found between higher temperatures and the infections $(r=0.4 ; \mathrm{p}<0.05)$ (Figure 1, panel B); however, no notable effects on Salmonella. Anatum infections have been associated with precipitation or humidity $(r<0.3 ; \mathrm{p}>0.05)$.

Detailed methods are described in the Appendix (https://wwwnc.cdc.gov/EID/article/26/12/200147-App1.pdf). We first reviewed the clinical and laboratory characteristics of 278 patients from 20152018. Most patients had acute gastroenteritis, whereas a few $(14 / 278,5 \%)$ had invasive diseases, such as bacteremia and sepsis. In terms of age distribution, the highest number of cases were in young children (Figure 1, panel C). Pediatric patients $(\mathrm{n}=169)$ had
Figure 1. Salmonella enterica serotype Anatum infection and antimicrobial resistance, Taiwan. A) Antimicrobial resistance of the Salmonella Anatum isolates collected in Chang Gung Memorial Hospital. B) Monthly case number (bar plot) and temperature (line). C) Age distribution of patients diagnosed during 2015-2018. significantly higher rates than adult patients $(\mathrm{n}=$ 109) for hospitalization $(79.2 \%$ vs. $55.0 \%$; $<<0.05)$, diarrhea $(89.9 \%$ vs. $68.8 \%$; $<<0.05)$, and fever $(89.2 \%$ vs. $58.1 \%$; $\mathrm{p}<0.05)$.

Multilocus sequence typing indicated that the entire collection of clinical Salmonella Anatum isolates belonged to sequence type 64 . We randomly selected 54 clinical isolates for WGS (Appendix Table 1). Both core genome multilocus sequence typing and whole-genome single-nucleotide polymorphism analyses, performed by using the BacWGSTdb database (9), further divided these isolates into 3 clades (Figure 2, panel A, B). Clades I and II were more closely related to each other; their most recent common ancestor occurred $>21$ years ago. 
Clade III was more distantly connected to these 2 clades. Typing based on PCR assay was performed on the unsequenced isolates. Clade I accounted for $95.6 \%(305 / 319)$ of all isolates, suggesting it was the cause of the outbreak. The isolates resistant to ceftriaxone, ciprofloxacin, or both clustered within clade I, whereas the isolates of clades II and III were more susceptible. Most of the clade I isolates harbored a 90-kb IncA/C plasmid carrying blaDHA-1 (encoding a class $C \beta$-lactamase) and $q \eta r B$ (conferring resistance to quinolones). A conjugation assay demonstrated that this plasmid conferred ceftriaxone and ciprofloxacin resistance. In addition, 31 $(9.7 \%)$ clinical isolates carried $b l a_{\mathrm{CMY}-2}$ which was located within a $>100-\mathrm{kb}$ IncI1 plasmid and also encoded a class $C \beta$-lactamase. These 31 isolates carried bla $a_{\text {DHA-1 }}$ simultaneously. In 11 of them, the $b l a_{\mathrm{DHA}-1}$-carrying and bla $a_{\mathrm{CMY}-2}$-carrying plasmids were fused into 1 large plasmid (Figure 2, panel C).

By comparing these findings against sequences in GenBank, we found nearly identical genomic sequences for isolates in the United Kingdom, the

Figure 2. Genomic analysis of the outbreak caused by Salmonella enterica serotype Anatum, Taiwan. A) Dated phylogeny for Salmonella Anatum clinical isolates and food and environmental isolates. All isolates were divided into 3 clades, shown at right. The nodes' colors represent the geo source; nodes with black rings were from meat or the environment, and the remainder were derived from the patients. The right heatmap represents the presence (in black) or absence (in gray) of key antimicrobial-resistance genes $\left(1\right.$, bla $a_{\mathrm{DHA}-1} ; 2$, qnrB $4 ; 3$, bla CMY-2 $\left._{\text {- }}\right)$. B) Minimal spanning tree based on alleles identified through core genome multilocus sequence typing. Dots with black circles represent food isolates; the others are clinical isolates. The collection date for the 6 US isolates in panel $B$ was missing in GenBank and therefore not included in panel A. Scale bar indicates 5 single nucleotide polymorphisms. C) Gene structure of multidrugresistant plasmids in Salmonella Anatum in Taiwan compared with international isolates. Two types of plasmids were identified in the clade I Salmonella. Anatum isolates in Taiwan. One carried bla ${ }_{\mathrm{CMY}-2}$, with its structure being shown by pSal-5091_CMY. A similar plasmid, pCMY2 (GenBank accession no. LC019731.1), is shown. The other carried bla $a_{\mathrm{DHA}-1}$; its structure is shown by pSal5091_DHA. International isolates shown in the figure, whose genomes also were downloaded from GenBank (Appendix Table 1, https://wwwnc.cdc.gov/EID/ article/26/12/20-0147-App1.pdf),

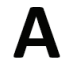

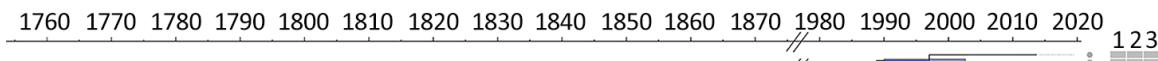

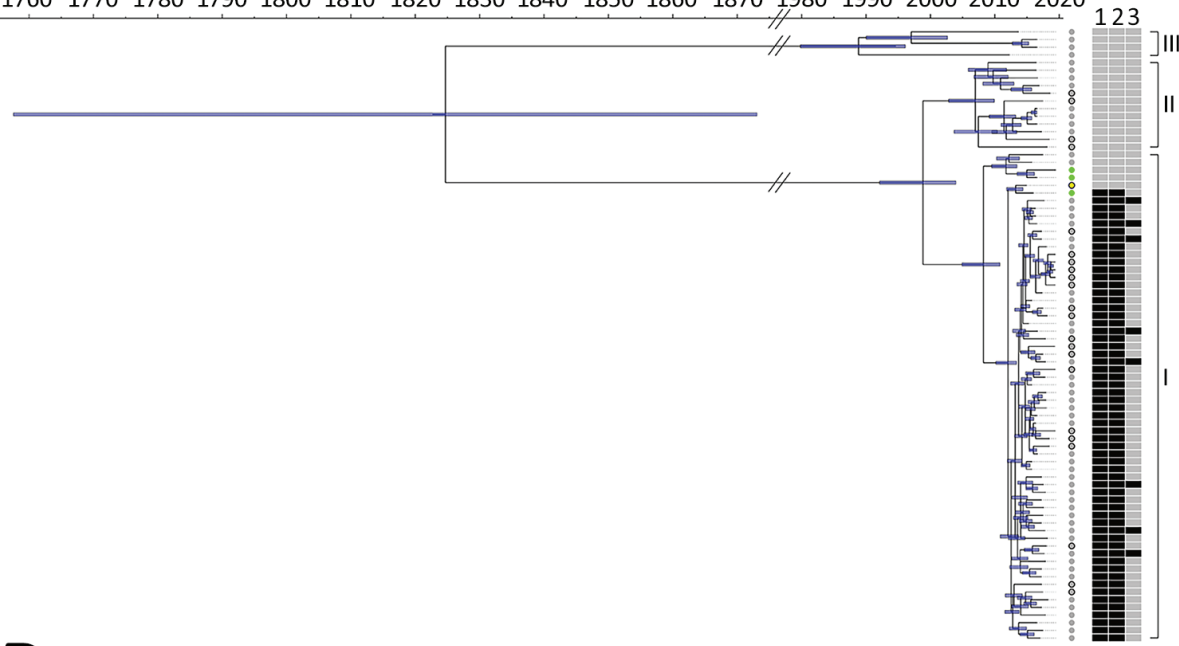

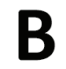

B 0

Clade I

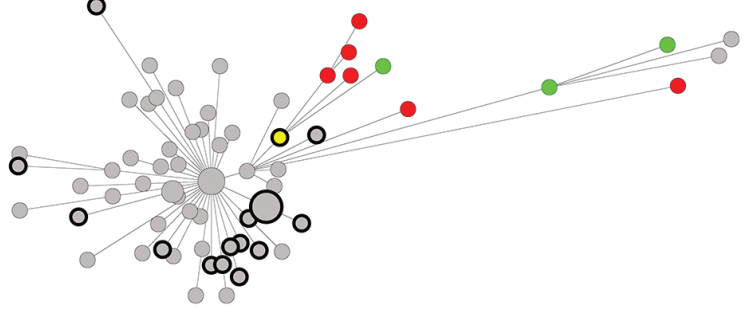

Clade II
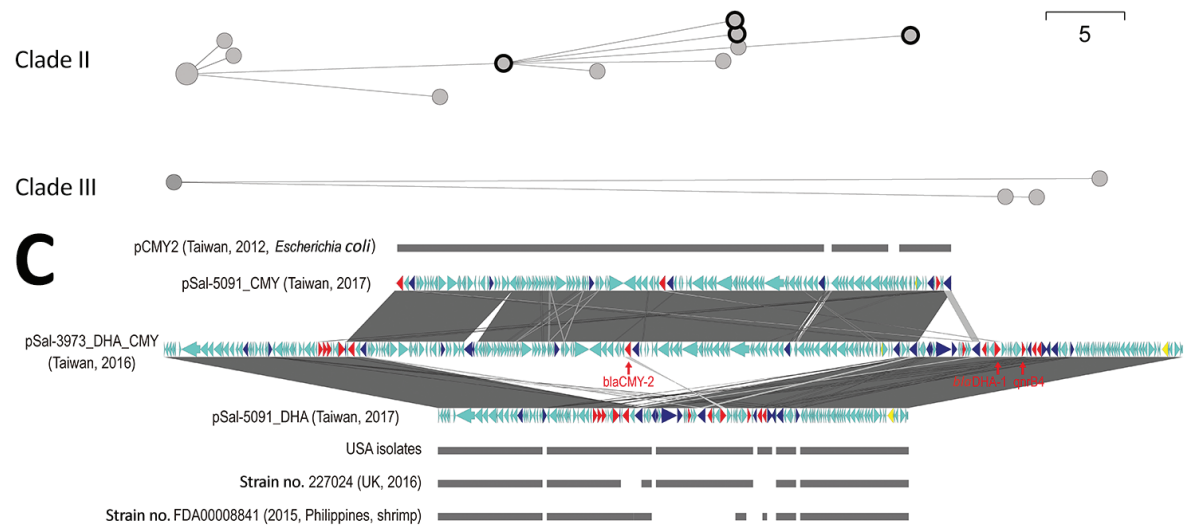
possess very similar plasmids. In certain isolates, the 2 plasmids can integrate into 1 large plasmid, with its structure shown by pSal-3973_DHA_CMY. Red genes represent antimicrobial-resistance genes; blue genes represent transposase/integrase genes; and yellow genes represent Inc-determinant genes. 
United States, and the Philippines. The collection time for these isolates also occurred during 20152019, which nearly coincided with the outbreak in Taiwan. These international Salmonella Anatum isolates also carried the $90-\mathrm{kb}$ IncA/C plasmid (Figure 2, panel A, C); therefore, they were likely ceftriaxone- and ciprofloxacin-resistant concomitantly. The only distinction of these international isolates was their lack of the $b l a_{\mathrm{CMY}-2}$-carrying plasmid. Accordingly, we speculated that the Salmonella Anatum clone had arrived in Taiwan through food trade and later acquired the $b l a_{\mathrm{CMY}-2}$-carrying plasmid.

To trace the source of Salmonella Anatum, we investigated food samples from supermarkets and traditional markets of 8 districts with high density of Salmonella patients in New Taipei City and Taoyuan City, Taiwan (Appendix Figure 1). A total of 11 Salmonella Anatum isolates were collected from pork, 4 from poultry, and 1 from beef in these regions (Appendix Table 2, Figure 1). WGS showed that they all belonged to clades I and II, providing strong evidence that raw meats were the outbreak vehicle. All 16 isolates harbored the $b l a_{\mathrm{DHA}-1}$-carrying IncA/C plasmid. Other Salmonella serovars also were detected in this investigation. The overall Salmonella isolation rate from retail meats was significantly higher in traditional markets than in the supermarkets $(p<0.001)$ (Appendix Table 3). In Taiwan, pork in the supermarkets is usually provided through the cold transportation chain, whereas for traditional markets pork is usually provided through the traditional chain, with notable differences. Temperatures were much lower in the cutting factory and butcher shop in the cold chain than in the traditional chain (Appendix Figure 2). Furthermore, pork was wrapped by plastic tissue and bags in the cold chain, but the traditional chain did not do any wrapping or packaging during transportation.

To clarify the contradictory findings that most infections occurred in young children even though pork is not a major food for infants, we conducted a questionnaire survey among parents of 20 infants $(<1$ year of age) with NTS infections and 80 parents of infants without (controls) (Appendix). Parents of the infected infants more often touched, rinsed, and cooked meat before feeding other foods to their infants (Appendix Table 4). Moreover, these parents were more willing to purchase meat from traditional markets rather than supermarkets. A possibility is that they bought meat from the traditional markets, then their frequent rinsing flushed the Salmonella on the surface of the meats, cutting boards and knives, and sinks, and finally onto fresh vegetables, fruit, and other ready-to-eat foods that were crosscontaminated and reached the infants through parents or other caregivers. This transmission mode is of particular importance in infants and has already been reported for other bacterial pathogens such as Yersinia enterocolitica (10).

\section{Conclusions}

Our study sought to describe an outbreak in Taiwan caused by a multidrug-resistant Salmonella Anatum clone. The questionnaire and supply-chain investigations we conducted found that the infection cases were closely associated with improper packaging during transportation and unhygienic food handling in the customers' kitchen. The high similarity of genomic sequence between the Taiwan isolates and international isolates indicates the global dissemination of this clone and highlights the public health value of multicountry sharing of epidemiologic, trace-back, microbiologic, genomic, and food trade data.

This study was financially supported by the National Natural Science Foundation, China (grant no. 31670132); the Chang Gung Memorial Hospital, Taiwan (grant nos. CRRPG3F0084, CMRPG3D1721-3, CMRPG3G1451-3, and CMRPG3E1371-3); and the Ministry of Health and Welfare, Taiwan (grant nos. MOHW106-CDC-C-114113702, MOHW107-CDC-C-114-123505, and MOHW108CDC-C-114-133505).

\section{About the Author}

Dr. Feng is an associate professor at the Institute for Translational Medicine, Zhejiang University School of Medicine, Hangzhou, China. His research interests are the epidemiology, genomics, and drug-resistance mechanisms of nontyphoidal Salmonella.

\section{References}

1. Chen HM, Wang Y, Su LH, Chiu CH. Nontyphoid Salmonella infection: microbiology, clinical features, and antimicrobial therapy. Pediatr Neonatol. 2013;54:147-52. https://doi.org/ 10.1016/j.pedneo.2013.01.010

2. Feng Y, Chang YJ, Fang SH, Su LH, Li HC, Yang HP, et al. Emergence and evolution of high-level cephalosporinresistant Salmonella Goldcoast in Northern Taiwan. Open Forum Infect Dis. 2019;6:ofz447. https:/ / doi.org/10.1093/ ofid/ofz447

3. Qi X, Li P, Xu X, Yuan Y, Bu S, Lin D. Epidemiological and molecular investigations on Salmonella responsible for gastrointestinal infections in the southwest of Shanghai from 1998 to 2017. Front Microbiol. 2019;10:2025. https:// doi.org/10.3389/fmicb.2019.02025

4. Jean SS, Lu MC, Shi ZY, Tseng SH, Wu TS, Lu PL, et al. In vitro activity of ceftazidime-avibactam, ceftolozanetazobactam, and other comparable agents against clinically important Gram-negative bacilli: results from the 2017 
Surveillance of Multicenter Antimicrobial Resistance in Taiwan (SMART). Infect Drug Resist. 2018;11:1983-92. https:/ / doi.org/10.2147/IDR.S175679

5. Zhan Z, Xu X, Gu Z, Meng J, Wufuer X, Wang M, et al. Molecular epidemiology and antimicrobial resistance of invasive non-typhoidal Salmonella in China, 2007-2016. Infect Drug Resist. 2019;12:2885-97. https:// doi.org/10.2147/ IDR.S210961

6. Chiou CS, Hong YP, Liao YS, Wang YW, Tu YH, Chen BH, et al. New multidrug-resistant Salmonella enterica serovar Anatum clone, Taiwan, 2015-2017. Emerg Infect Dis. 2019;25:144-7. https://doi.org/10.3201/eid2501.181103

7. Ruan Z, Yu Y, Feng Y. The global dissemination of bacterial infections necessitates the study of reverse genomic epidemiology. Brief Bioinform. 2020;21:741-50. https:// doi.org/10.1093/bib/bbz010

8. Nadon C, Van Walle I, Gerner-Smidt P, Campos J, Chinen I, Concepcion-Acevedo J, et al.; FWD-NEXT Expert
Panel. PulseNet International: vision for the implementation of whole genome sequencing (WGS) for global food-borne disease surveillance. Euro Surveill. 2017;22:30544. https:/ / doi.org/10.2807/1560-7917.ES.2017.22.23.30544

9. Ruan Z, Feng Y. BacWGSTdb, a database for genotyping and source tracking bacterial pathogens. Nucleic Acids Res. 2016;44(D1):D682-7. https://doi.org/10.1093/nar/ gkv1004

10. Lee LA, Gerber AR, Lonsway DR, Smith JD, Carter GP, Puhr ND, et al. Yersinia enterocolitica O:3 infections in infants and children, associated with the household preparation of chitterlings. N Engl J Med. 1990;322:984-7. https://doi.org/10.1056/NEJM199004053221407

Address for correspondence: Cheng-Hsun Chiu, Department of Pediatrics, Chang Gung Memorial Hospital, No. 5, Fu-Hsin St, Kweishan 333, Taoyuan, Taiwan; email: chchiu@adm.cgmh.org.tw

\title{
etymologia
}

\section{Salmonella [sal"mo-nel'ə]}

\author{
Daniel F. M. Monte, Fábio P. Sellera
}

$\mathrm{N}^{2}$ amed in honor of Daniel Elmer Salmon, an American veterinary pathologist, Salmonella is a genus of motile, gram-negative bacillus, nonspore-forming, aerobic to facultatively anaerobic bacteria of the family Enterobacteriaceae. In 1880, Karl Joseph Eberth was the first to observe Salmonella from specimens of patients with typhoid fever (from the Greek typhōdes [like smoke; delirious]), which was formerly called Eberthella typhosa in his tribute. In 1884, Georg Gaffky successfully isolated this bacillus (later described as Salmonella Typhi) from patients with typhoid fever, confirming Eberth's findings. Shortly afterward, Salmon and his assistant Theobald Smith, an American bacteriologist, isolated Salmonella Choleraesuis from swine, incorrectly assuming that this germ was the causative agent of hog cholera. Later, Joseph Lignières, a French bacteriologist, proposed the genus name Salmonella in recognition of Salmon's efforts.

With a complicated taxonomy, the genus Salmonella is currently classified into 2 species (S. enterica and S. bongori), encompassing 2,659 serotypes based on somatic $\mathrm{O}$ and $\mathrm{H}$ flagellar antigens as specified in the Kauffmann-White-Le Minor scheme. S. enterica is divided into 6 subspecies: enterica, salamae, arizonae, diarizonae, houtenae, and indica. Arguably, this zoonotic pathogen remains one of the most pressing global concerns. It causes a spectrum of diseases in several hosts, and there is much to be learned and deciphered about its continuous evolution.

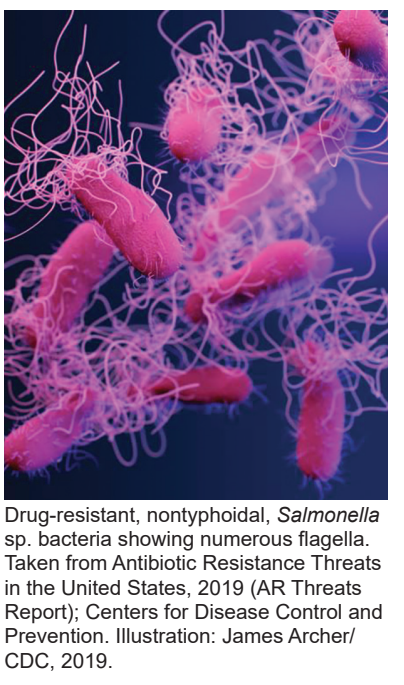

\section{Sources}

1. Dorland's Illustrated Medical Dictionary. 32nd ed. Philadelphia: Elsevier Saunders; 2012.

2. Gossner CM, Le Hello S, de Jong B, Rolfhamre P, Faensen D, Weill FX, et al. Around the world in 1,475 Salmonella geo-serotypes [Another Dimension]. Emerg Infect Dis. 2016;22:1298-302. https:/ / doi.org/10.3201/ eid2207.141678

3. Issenhuth-Jeanjean $S$, Roggentin $P$, Mikoleit $M$, Guibourdenche M, de Pinna E, Nair S, et al. Supplement

2008-2010 (no. 48) to the White-Kauffmann-Le Minor scheme. Res Microbiol. 2014;165:526-30. https://doi. org/10.1016/j.resmic.2014.07.004

4. Salmon DE. The discovery of the germ of swine-plague. Science. 1884;3:155-8. https:/ / doi.org/10.1126/ science. ns-3. $=53.155$

5. Su LH, Chiu CH. Salmonella: clinical importance and evolution of nomenclature. Chang Gung Med J. 2007;30:210-9.

\section{Author affiliations: University of São Paulo, São Paulo, Brazil}

Address for correspondence: Daniel F. M. Monte, Department of Food and Experimental Nutrition, Faculty of Pharmaceutical Sciences, University of São Paulo, São Paulo-SP 05508-900, Brazil; email: monte_dfm@usp.br 